\title{
Endemic non-venereal syphilis (bejel) in Saudi
}

\section{Arabia}

\author{
J L PACE* AND G W CSONKA†
}

From the *Department of Dermatology, King Abdul Aziz Hospital, Tabuk, Saudi Arabia, and the †John Hunter Clinic, St Stephen's Hospital, Chelsea, London

SUMMARY A total of 2515 people attending a large military hospital in Saudi Arabia was studied clinically, serologically, and (when appropriate) radiologically for evidence of treponematosis. The indications are that non-venereal endemic syphilis (bejel) is prevalent among the nomadic communities living in rural areas. In contrast, venereal syphilis is much less common, and is found almost exclusively in urban populations. Some of the high risk regions for bejel have been identified, and many people from these locations complained of persistent pain in the legs, which was often associated with radiological evidence of osteoperiostitis of the long bones. Bejel also seems to have become clinically "attenuated" within the last 30 years, with the majority of seroreactors having latent disease. A hypothesis suggesting a reason for this change is put forward, and ways of controlling the infection are outlined.

\section{Introduction}

Endemic syphilis, which is called bejel in the Middle East, is a non-venereal treponematosis that has its onset in early childhood and is transmitted from child to child by close skin contact, kissing, and possibly by fomites such as communal drinking vessels. It is caused by an organism morphologically indistinguishable from Treponema pallidum, which is easily seen on dark field microscopy of exudate from early lesions of the mouth and skin. There is no evidence of congenital transmission, or that it affects the cardiovascular or nervous systems. In its classic form bejel is easily recognised as a clinical entity, with initial infectious mucous patches in the mouth followed by a generalised non-irritant rash, which is much more pronounced and persistent than that seen in venereal syphilis. A proportion of patients develop a late clinical stage with gummata of the skin and bones, which sometimes progresses to destructive lesions especially of the nasal septum and palate. Painful osteoperiostitis of the tibia and fibula is common. Late latent bejel is now the predominant form of the disease in later years. Treatment with penicillin gives excellent results in early lesions and gummata, but its effect is more variable when bone is

Address for reprints: Dr G W Csonka, The John Hunter Clinic, St
Stephen's Hospital, Fulham Road, Chelsea, London SW10 9TH

Accepted for publication 13 January 1984 affected. The classic form was first reported from Syria and later in Iraq. ${ }^{1-3}$ It has been found in Yugoslavia, where it has now been completely eradicated. ${ }^{4}$ It is still present in parts of Africa and South East Asia, especially in remote rural areas with limited access to static health services.

This once florid disease has changed appreciably within a generation, having been replaced by a milder form in which the number, severity, and duration of both early and late lesions are reduced. The reason for this change is not clear. In a recent report by the World Health Organisation (WHO) the patchy distribution of areas where non-venereal syphilis persists is linked with inadequate medical services and also with the increase of atypical and latent cases, the importance of which is not appreciated by clinicians unfamiliar with this condition. ${ }^{5}$ Serological tests for venereal syphilis give positive results. A simple serological survey of suspected rural communities would thus provide essential epidemiological data to map the location of foci of endemic syphilis and form a basis for introducing control measures.

The presence of bejel in Saudi Arabia was first reported in 1954 by a team from the WHO and was confirmed in 1979.6-8 Such studies are important as mistaking bejel for venereal syphilis can have catastrophic social consequences. In this study, using clinical, serological, and radiological examination for treponematosis, we compared nomadic people attending one large hospital from a country wide catchment area with those born and bred in town. 


\section{Patients and methods}

In a first (retrospective) study the medical records of 105 patients seen at Tabuk Military Hospital between 1976 and 1980 who had been diagnosed as suffering from venereal syphilis were assessed according to whether they were nomads or were born and lived in cities. In a second (prospective) investigation 527 women attending the antenatal clinic and 263 of their husbands were examined clinically and serologically and compared according to whether they were nomads or town people. In a third (prospective) study 1620 nomadic patients coming from all parts of the country to attend the primary care unit of the hospital were similarly screened.

Patients found to have venereal syphilis received 2.4 MU benzathine penicillin by intramuscular injection once a week for three weeks. Patients thought to have bejel were given a single intramuscular injection of $1 \cdot 2 \mathrm{MU}$ benzathine penicillin. The Venereal Diseases Research Laboratory (VDRL) and Treponema pallidum haemagglutination
(TPHA) tests were used routinely; in cases of doubt the fluorescent treponemal antibody absorbed (FTAABS) test was added. Radiographs of the long bones of the legs and occasionally the forearms and facial bones were taken when appropriate. In all but 200 of the third study group, radiology of the tibia and fibula was part of the routine investigation.

\section{Results}

Table I shows the results of the retrospective study, and table II gives those from the antenatal clinic. No cases of congenital syphilis were seen in infants born to untreated seropositive mothers. Table III compares the distribution of seroreactors among the 1620 nomadic patients according to sex and shows a preponderance of women. Most of the patients in this group were between 15 and 35 . The domicile of seroreactors among the 1620 nomads is indicated in the sketch map (fig 1), from which it may be seen that there is clustering in two areas.

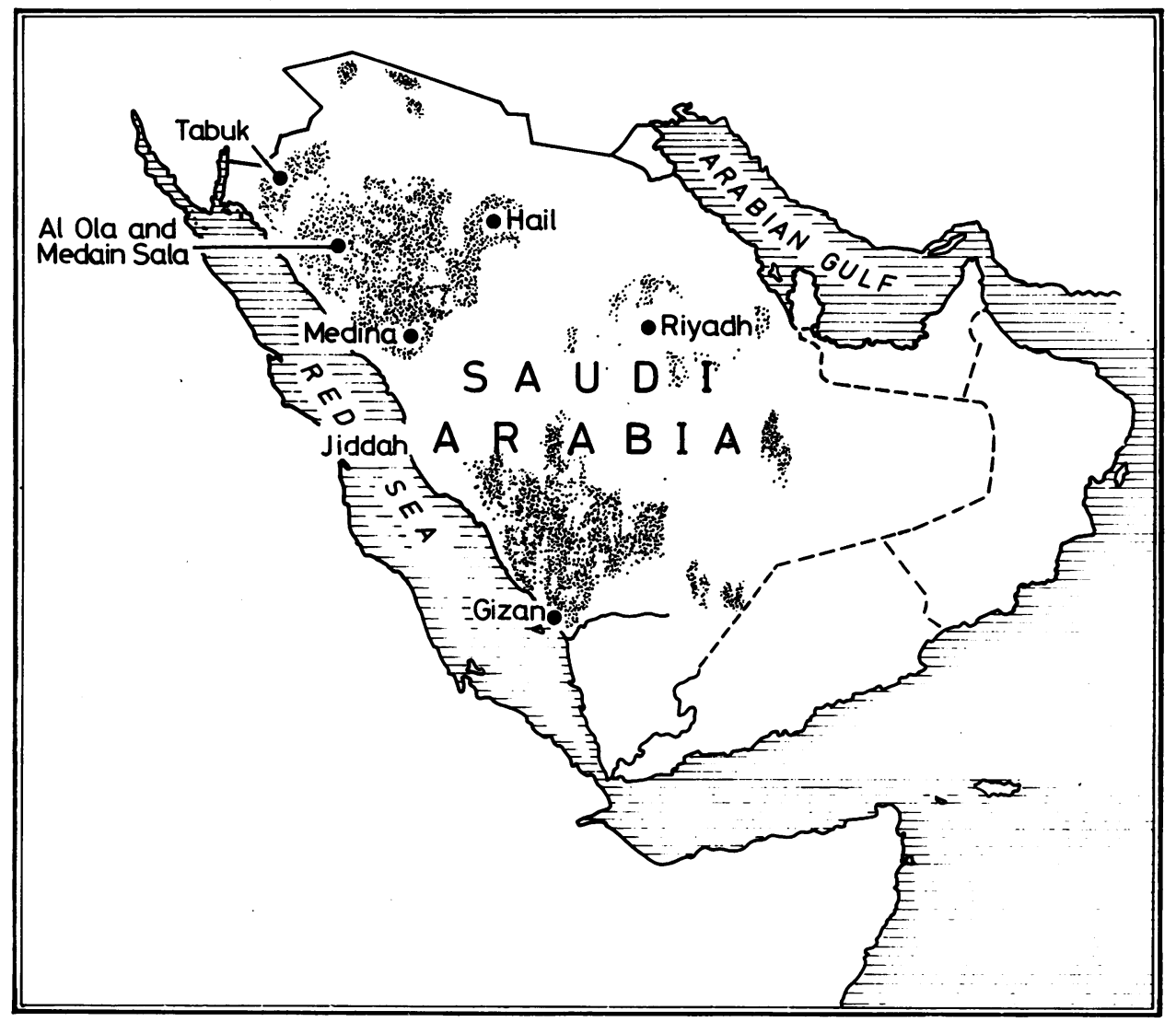

FIG 1 Sketch map of Saudi Arabia with stippling showing the areas in which the 276 nomadic seroreactors live. 
TABLE I Retrospective study of 105 seropositive patients who had attended Abdul Aziz Hospital, Tabuk (1976-80) and been diagnosed as having syphilis

\begin{tabular}{lll}
\hline & Lifestyle & \\
\cline { 2 - 3 } $\begin{array}{l}\text { Diagnosis according to } \\
\text { clinical evidence }\end{array}$ & Nomads & City dwellers \\
\hline $\begin{array}{l}\text { Probably endemic } \\
\text { non-venereal syphilis }\end{array}$ & $99(94 \cdot 3 \%)$ & 0 \\
\begin{tabular}{l} 
Venereal syphilis \\
\hline
\end{tabular} & 0 & $6(5 \cdot 7 \%)$ \\
\hline
\end{tabular}

There was radiological evidence of osteoperiostitis of the tibia or the fibula, or both, in $15 \%$ of seroreactors, which was comparable with that seen by one of us (GWC) in Iraq 30 years ago (fig 2). Most of the patients with such radiological abnormalities complained of pain in the legs, which worsened at night, was sometimes severe enough to interfere with work, and on occasion curtailed all activities. Radiological evidence of osteoperiostitis of the ulna and radius was sometimes present but was possibly underestimated as there was no accompanying discomfort.

TABLE II Results of serological (FTA-ABS) tests in 790 Saudi nationals attending the antenatal clinic in Tabuk

\begin{tabular}{lcc}
\hline Lifestyle & No & No (\%) positive \\
\hline City dwellers & 609 & $2(0 \cdot 3)$ \\
Nomads & 181 & $35(19 \cdot 3)$ \\
\hline
\end{tabular}

Other late lesions were seen rarely, and included gummata of the skin, the palate, and the nasal septum. One middle aged Bedouin woman with longstanding hoarseness was found to have granulomatous lesions of the larynx and was diagnosed as having tuberculous laryngitis but failed to respond to antitubercular treatment. The results of her VDRL and FTA-ABS tests were positive, and the lesions cleared promptly after treatment with penicillin. One remarkable patient presented with extreme destruction of the face and naso-pharynx (figs 3 and 4 ) and is at present undergoing plastic repair operations.

\section{Discussion}

This study shows that in Saudi Arabia we are dealing with two different populations at risk of treponemal

TABLE III Results of serological screening for treponematosis in 1620 nomads attending the primary care unit in Tabuk

\begin{tabular}{lll}
\hline & No & No (\%) positive \\
\hline Men & 9972 & $143(14 \cdot 7)$ \\
Women & 648 & $133(20 \cdot 5)$ \\
Total & 1620 & $276(17)$ \\
\hline
\end{tabular}

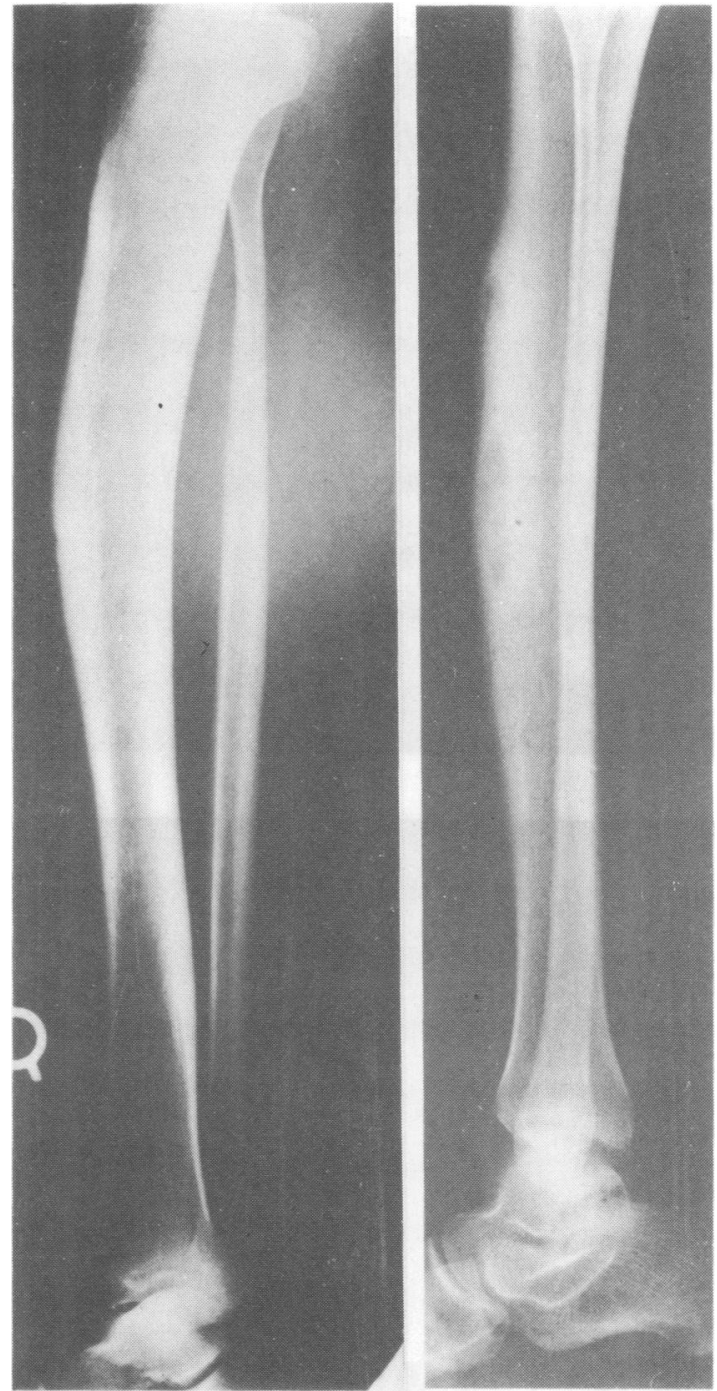

FIG 2 Osteoperiostitis of tibia and fibula, Saudi Arabia, 1981 (left); osteoperiostitis with some osteolytic changes involving mainly the tibia, Iraq, 1951 (right).

infection. These are the nomadic Bedouins who have a high incidence of endemic non-venereal syphilis, and the people born and bred in towns who no longer acquire endemic syphilis but may develop venereal syphilis because the opportunity of exposure is much greater. Our findings suggest that bejel accounts for most positive serological reactions found in nomadic communities and probably outweighs venereal syphilis in the population as a whole.

The absence of very young children in our study population may partly explain the absence of early 

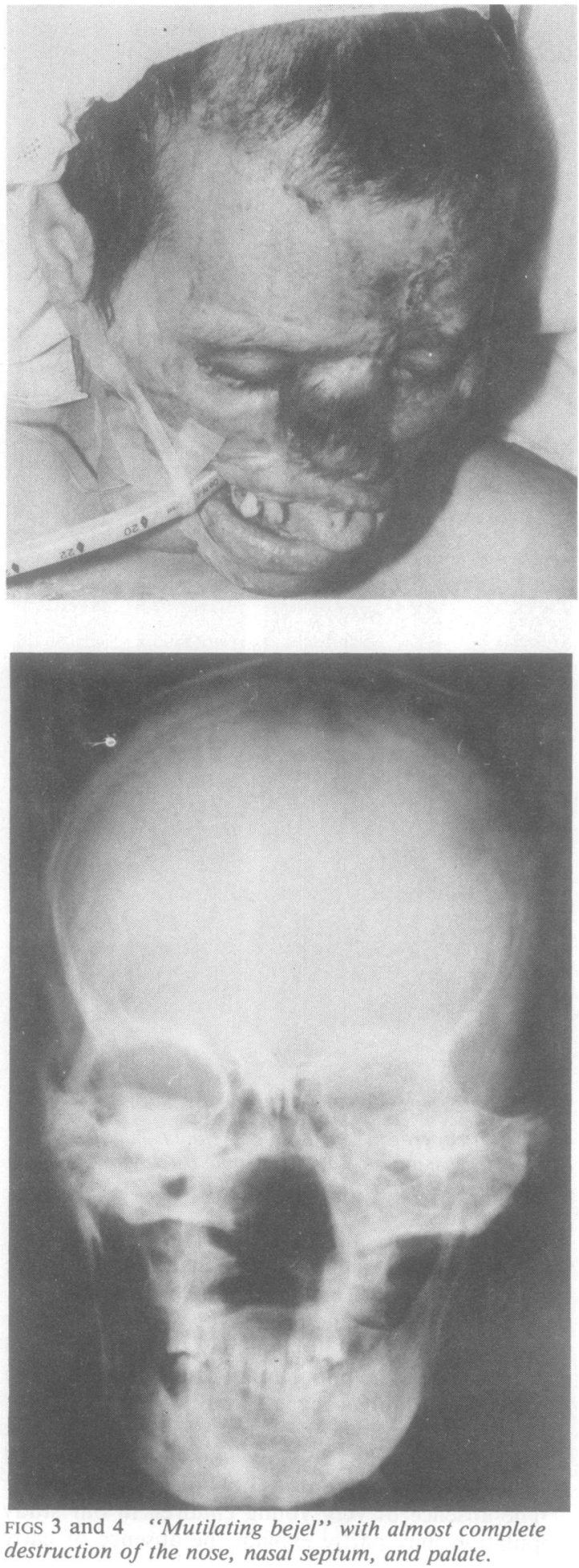

mucous membrane lesions of the mouth and the generalised rash associated with bejel. From our experience in Iraq during the height of the bejel epidemic, however, skin lesions often persisted into adolescence. There therefore appears to be a genuine decrease in such clinically obvious lesions. This phenomenon of "attenuated" treponemal infection has also recently been reported from other parts of the world with different types of endemic syphilis. ${ }^{5}$

The most common late manifestation noted by us was painful osteoperiostitis of the legs affecting mainly the tibia and fibura. It was of interest that many patients themselves associated the pain in the legs with bejel that they remembered having had in their childhood. Penicillin was given to patients whose bones were affected, but it is too early to assess its efficacy. Past observations in Iraq indicate that while it halts progression of the disease, its influence on pain is variable, and if radiological changes have been present for more than a year they usually prove permanent. ${ }^{3}$ Destructive lesions of the palate and nasal septum are distressing features of untreated bejel as they are associated with difficulties in speech and eating and are disfiguring. The extreme case illustrated here is very rare but a similar case has been reported in an adolescent boy with bejel in Iraq. ${ }^{11}$ Apart from the common osteoperiostitis of the long bones, the more dramatic late destructive lesions, like the early infectious lesions, also appear to have become infrequent. We suggest that the early persistent lesions were sustained by frequent superinfection and such repeated contacts have become uncommon with the improvement of hygiene. Thus the number, severity, and duration of early lesions have declined. There is evidence that in patients with past untreated endemic non-venereal syphilis, reexposure to new infection many years later precipitates late lesions. ${ }^{4}$ Thus the chances of developing late lesions diminish with the decrease in re-exposure.

From our experience we think that the time has come for field studies to be started in Saudi Arabia, as we have identified several high risk communities. The inhabitants of these areas should be systematically screened clinically and serologically, with special reference to children aged under 12 , to establish the prevalence and clinical range of the disease under present circumstances. If our assumption from the available evidence is correct we would expect that $5 \%$ or less of people living in endemic areas of Saudi Arabia will have early active lesions. Such patients and their immediate contacts should be treated. The treatment recommended by the WHO consists of a single injection of $1.2 \mathrm{MU}$ benzathine penicillin for adults and $0.6 \mathrm{MU}$ for children aged under 10 . In the event of there being more than $5 \%$ of such patients in a community, they, 
their contacts, and all children aged under 10 should receive treatment (selective mass treatment). ${ }^{5}$ Yearly surveys are essential until proof of eradication has been obtained or the rural health services can cope.

A factor that was not important in the past is the increasing mobility of people, which can lead to the spread of the infection across frontiers. Thus, cooperation of all countries adjacent to an area with endemic syphilis is necessary to limit such spread. At the same time as the field studies are proceeding, nomads attending the major hospitals in the country should be routinely screened for endemic syphilis by the VDRL and TPHA tests, and the results entered on a master map. In this way one can expect fairly soon to have a comprehensive picture of the main foci of bejel. These data can be constantly updated and should prove useful in campaigns for screening and treatment. Following such campaigns it is likely that the infection will be controlled or even eradicated in Saudi Arabia.

It might be argued that if improving hygiene is essential for eliminating non-venereal syphilis, then raising the standard of hygiene should take priority over all else. There may be some truth in this, but the intelligent use of penicillin now can speed the process of control, and the opportunities given by treatment campaigns can in any case be used to improve hygiene. There is yet another consideration to support the use of penicillin without delay: although there is as yet no evidence of the emergence of treponemes resistant to penicillin in Saudi Arabia, this may not always be the case. Every endeavour should therefore be made to control endemic syphilis while the major available antibiotic is still effective.

Finally, some observers think that eliminating endemic non-venereal syphilis at present might remove some immunological protection against venereal syphilis, which is a potentially much more serious condition. We believe that endemic syphilis should be eradicated as it can cause avoidable chronic ill health, and venereal syphilis should be treated as and when it arises.

\section{References}

1. Hudson EH. Treponematosis among the Bedouin Arabs of the Syrian desert. Naval Medical Bulletin 1928;26:817-24.

2. Hudson EH. Bejel: the endemic syphilis of the Euphrates Arab. Trans $R$ Soc Trop Med Hyg 1937;31:9-46.

3. Csonka GW. Clinical aspects of bejel. Br J Vener Dis 1953;29: 95-103.

4. Grin EI. Epidemiology and control of endemic syphilis. WHO Monogr Ser 1953 (No 11) 1-74.

5. Treponemal infections. WHO Tech Rep Ser 1982;674: 16-20.

6. Ghouroury AA. The syphilis problem in Asir province, Saudi Arabia. Bull WHO 1954; 10:691-702.

7. Sebai ZA, Baker TD. Endemic syphilis (bejel) in Saudi Arabia. Ain Shams Medical Journal 1979; 30: 13-7.

8. Pace JL. Treponematoses in Arabia. Saudi Medical Journal 1983;4:211-20.

9. Chowdury MNH, Jamjoom GA, Pareek SS, Mahgoub ES Treponemal diseases in Riyadh, Saudi Arabia. Saudi Medical Journal 1982:3:31-40.

10. Fatani M, Asfahani A, Aman H, Saleem M, Kuweity K. Is syphilis a problem in Jeddah? Proceedings of the VI Saudi Medical Conference. Jeddah: King Abdul Aziz University Press, 1981:78-9.

11. Jones LG. "Mutilating" bejel. Br J Vener Dis 1953; 29: 104-6. 